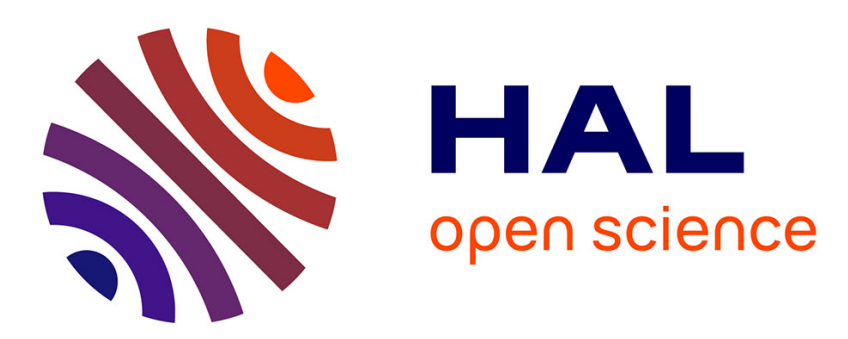

\title{
Product Approximations for Solutions to a Class of Evolution Equations in Hilbert Space
}

\author{
Pierre-A. Vuillermot, Walter Wreszinski
}

\section{To cite this version:}

Pierre-A. Vuillermot, Walter Wreszinski. Product Approximations for Solutions to a Class of Evolution Equations in Hilbert Space. Portugaliae Mathematica, 2011, 68 (3), pp.317-343. 10.4171/PM/1894 . hal-00536627

\section{HAL Id: hal-00536627 https://hal.science/hal-00536627}

Submitted on 16 Nov 2010

HAL is a multi-disciplinary open access archive for the deposit and dissemination of scientific research documents, whether they are published or not. The documents may come from teaching and research institutions in France or abroad, or from public or private research centers.
L'archive ouverte pluridisciplinaire HAL, est destinée au dépôt et à la diffusion de documents scientifiques de niveau recherche, publiés ou non, émanant des établissements d'enseignement et de recherche français ou étrangers, des laboratoires publics ou privés. 


\title{
Product Approximations for Solutions to a Class of Evolution Equations in Hilbert Space
}

\author{
Pierre-A. Vuillermot* and Walter F. Wreszinski** \\ UMR-CNRS 7502, Institut Élie Cartan, Nancy* \\ Departamento de Física Matemática, Universidade de São Paulo**
}

\begin{abstract}
In this article we prove approximation formulae for a class of unitary evolution operators $U(t, s)_{s, t \in[0, T]}$ associated with linear non-autonomous evolution equations of Schrödinger type defined in a Hilbert space $\mathcal{H}$. An important feature of the equations we consider is that both the corresponding self-adjoint generators and their domains may depend explicitly on time, whereas the associated quadratic form domains may not. Furthermore the evolution operators we are interested in satisfy the equations in a weak sense. Under such conditions the approximation formulae we prove for $U(t, s)$ involve weak operator limits of products of suitable approximating functions taking values in $\mathcal{L}(\mathcal{H})$, the algebra of all linear bounded operators on $\mathcal{H}$. Our results may be relevant to the numerical analysis of $U(t, s)$ and we illustrate them by considering two evolution problems in quantum mechanics.
\end{abstract}

\section{Introduction and Outline}

Let $\mathcal{H}$ be an arbitrary complex Hilbert space and let $\mathcal{L}(\mathcal{H})$ be the algebra of all bounded linear operators defined on $\mathcal{H}$. Our purpose in this article is to prove approximation formulae for the solutions to initial-value problems of the form

$$
\begin{aligned}
i \frac{d u(t)}{d t} & =H(t) u(t), \quad 0 \leq s<t \leq T, \\
u(s) & =v
\end{aligned}
$$

where the $H(t)$ 's are given self-adjoint and positive operators in $\mathcal{H}$, with $T \in$ $(0,+\infty)$ arbitrary. More specifically, assuming there exists a unitary evolution system $U_{H}(t, s)_{s, t \in[0, T]}$ on $\mathcal{H}$ that solves (1) in a suitably weak sense, we display a large one-parameter family of functions $F_{t}: \mathbb{R}^{+} \mapsto \mathcal{L}(\mathcal{H})$ such that formulae of the form

$$
U_{H}(t, s)=\lim _{n \rightarrow+\infty} \prod_{\gamma=n-1}^{0} F_{s+\frac{\gamma}{n}(t-s)}\left(\frac{t-s}{n}\right)
$$


hold in the weak operator topology of $\mathcal{L}(\mathcal{H})$ for all $s, t \in[0, T]$ with $t \geq s$. We carry this out under hypotheses that allow the explicit time dependence of the domains of the $H(t)$ 's, whereas the associated quadratic form domains remain time-independent. The conditions we impose are more general than those used previously by various authors in the context of Schrödinger equations, who typically assume that the domains of the $H(t)$ 's are time-independent (see for instance [11], [20], and also [26] along with the references therein for the analysis of more general evolution equations). They are, in fact, related to the classic results of [15] and [21], which play a significant rôle in the sequel regarding the existence and various properties of unitary evolution systems $U(t, s)_{s, t \in[0, T]}$.

An important consequence of the theorem we state below is that among all the admissible functions $F_{t}$ there are the resolvent operators

$$
R_{t}(\tau):=(\mathbb{I}+i \tau H(t))^{-1}
$$

where $\mathbb{I}$ stands for the identity in $\mathcal{L}(\mathcal{H})$, and the $\mathcal{C}_{0}$-unitary semigroup

$$
S_{t}(\tau):=\exp [-i \tau H(t)] .
$$

This establishes the validity of the formulae

$$
\begin{aligned}
U_{H}(t, s) & =\lim _{n \rightarrow+\infty} \prod_{\gamma=n-1}^{0}\left(\mathbb{I}+i \frac{t-s}{n} H\left(s+\frac{\gamma}{n}(t-s)\right)\right)^{-1} \\
& =\lim _{n \rightarrow+\infty} \prod_{\gamma=n-1}^{0} \exp \left[-i \frac{t-s}{n} H\left(s+\frac{\gamma}{n}(t-s)\right)\right]
\end{aligned}
$$

under very general conditions. Furthermore, formulae such as (2) with the largest possible class of $F_{t}$ 's are also very useful in view of many applications since they constitute the theoretical basis of numerical algorithms intended to compute solutions to various differential problems, a theme thoroughly discussed in [6]. In particular, the resolvent approximation in (5) is typically related to the so-called Euler backward difference scheme.

We shall organize the remaining part of this article in the following way: in Section 2 we state our main result, which holds under three main hypotheses. In the first one we describe the topological and metric properties of the quadratic form domains we need to carry out our estimates, while in the other two we specify the class of unitary evolutions and of approximating functions for which (2) holds. In that section we also state a corollary where we establish the validity of (2) and (5) when $H(t)$ splits as

$$
H(t)=H_{0} \dot{+} V(t)
$$

in the sense of quadratic forms, with $H_{0}$ and $V(t)$ self-adjoint operators in $\mathcal{H}, H_{0}$ positive and time-independent and $V(t)$ subordinated to $H_{0}$ in some sense. This is of course one of the typical situations encountered in the realm of quantum mechanics, and the existence of the unitary evolution $U_{H_{0}+V}(t, s)_{s, t \in[0, T]}$ we 
need there is garanteed by some of the results in [15] and [21]. In this last case we also note that (5) does not take the form of the usual Trotter product formulae in spite of the decomposition (6), a point we shall briefly discuss at the end of Sections 2 and 3. We devote Section 3 to the proofs of our results, which rest on duality arguments involving the quadratic form domains associated with the $H(t)$ 's, and on a natural generalization of the methods we developed in [24] and [25] for the investigation of parabolic evolution equations. In Section 4 we illustrate our results by means of two examples. The first one relates to the evolution of a particle in one space dimension under the influence of a finite number of time-dependent point interactions, a special case of a model originally introduced in [8] and recently revisited in [18] and [19], while the second one describes the motion of a quantum particle in three-dimensional Euclidean space subjected to a so-called time-dependent Rollnik potential.

\section{Statement of the Results}

In the sequel we write $(.,$.$) for the inner product in \mathcal{H}$ and $\|$.$\| for the corre-$ sponding induced norm. We also denote by $\|\cdot\|_{\infty}$ the usual supremum-norm in $\mathcal{L}(\mathcal{H})$.

For an arbitrary $T \in(0,+\infty)$ and for each $t \in[0, T]$ we consider initialvalue problems of the form (1), where the $H(t)$ 's are self-adjoint and positive operators defined on dense domains $\mathcal{D}(H(t))$ which may depend explicitly on $t$. Let $Q(t)_{t \in[0, T]}$ be the one-parameter family of closed and Hermitian sesquilinear forms associated with the $H(t)$ 's through the second representation theorem for quadratic forms, densely defined on the domain $\mathcal{D}_{Q}:=\mathcal{D}\left(H(t)^{\frac{1}{2}}\right)$ (see, for instance, [14] for a discussion of this theorem). In what follows we assume that $\mathcal{D}_{Q}$ is independent of $t$ and that $Q(t)$ satisfies the positivity condition

$$
Q(t)[v, v] \geq c\|v\|^{2}
$$

for some constant $c \in(0,+\infty)$ uniformly in $t$ for every $v \in \mathcal{D}_{Q}$. As is well known, this allows one to endow $\mathcal{D}_{Q}$ with the natural unitary structure defined from the inner products

$$
(v, w)_{Q, t}:=Q(t)[v, w]=\left(H(t)^{\frac{1}{2}} v, H(t)^{\frac{1}{2}} w\right)
$$

and we write $\mathcal{H}_{Q, t}$ for the corresponding Hilbert spaces equipped with the induced norms

$$
\|v\|_{Q, t}:=\left\|H(t)^{\frac{1}{2}} v\right\| \text {. }
$$

Let $\mathcal{H}_{Q, t}^{*}$ be the adjoint space of $\mathcal{H}_{Q, t}$ endowed with the usual norm

$$
\|w\|_{Q, t, *}:=\sup _{0 \neq v \in \mathcal{H}_{Q, t}} \frac{\left|\langle w, v\rangle_{*}\right|}{\|v\|_{Q, t}}
$$


where $\langle., .\rangle_{*}$ stands for the duality bracket between $\mathcal{H}_{Q, t}$ and $\mathcal{H}_{Q, t}^{*}$. We infer from (7), (9) and (10) that the continuous embeddings

$$
\mathcal{H}_{Q, t} \mapsto \mathcal{H} \mapsto \mathcal{H}_{Q, t}^{*}
$$

hold provided we identify $\mathcal{H}$ with its adjoint space in the usual manner by means of Riesz's lemma. In this setting the vector space $\mathcal{D}_{Q}$ is dense in $\mathcal{H}_{Q, t}^{*}$ with respect to (10) (see, for instance, [15], [16] and [22] for other typical constructions of this kind). Moreover, the two embedding constants relative to (11) are independent of $t$ and furthermore we may write (10) as

$$
\|w\|_{Q, t, *}=\left\|H(t)^{-\frac{1}{2}} w\right\|,
$$

where $H(t)^{-\frac{1}{2}}$ is the extension by continuity to $\mathcal{H}_{Q, t}^{*}$ of the corresponding operator on $\mathcal{H}$. Thus, the $\mathcal{H}_{Q, t}^{*}$ 's inherit a Hilbert space structure as well with respect to the inner products

$$
(v, w)_{Q, t, *}:=\left(H(t)^{-\frac{1}{2}} v, H(t)^{-\frac{1}{2}} w\right) .
$$

It is worth recalling here that for all $s, t \in[0, T]$ the norms $\|\cdot\|_{Q, s}$ and $\|\cdot\|_{Q, t}$ are mutually equivalent since the linear operators $H(s)^{\frac{1}{2}} H(t)^{-\frac{1}{2}}$ are bounded on $\mathcal{H}$, a simple consequence of (7), the time-independence of $\mathcal{D}_{Q}$ and the closed graph theorem. This implies in particular that the spaces $\mathcal{H}_{Q, t}$ are all algebraically and topologically identical, as are the spaces $\mathcal{H}_{Q, t}^{*}$. Therefore, from now on we write $\mathcal{H}_{Q}$ and $\mathcal{H}_{Q}^{*}$ for these spaces, respectively, whenever their metric properties are not directly involved.

In view of the applications of Section 4 we have now to impose more stringent conditions on the family $Q(t)_{t \in[0, T]}$. Indeed, we assume that the following hypothesis is valid:

(Q) There exist an additional, fixed norm $\|\cdot\|_{+}$on $\mathcal{H}_{Q}$ and a constant $c \in$ $[1,+\infty)$ such that the inequalities

$$
c^{-1}\|v\|_{+}^{2} \leq Q(t)[v, v] \leq c\|v\|_{+}^{2}
$$

hold for each $t \in[0, T]$ and every $v \in \mathcal{H}_{Q}$. Moreover, there exists a constant $c^{*} \in(0,+\infty)$ such that the Lipschitz continuity estimate

$$
|Q(t)[v, v]-Q(s)[v, v]| \leq c^{*}|t-s|\|v\|_{+}^{2}
$$

holds for all $s, t \in[0, T]$ and every $v \in \mathcal{H}_{Q}$.

The existence of $\|\cdot\|_{+}$on $\mathcal{H}_{Q}$ implies the existence of an additional fixed norm $\|\cdot\|_{-}$on $\mathcal{H}_{Q}^{*}$, namely,

$$
\|w\|_{-}:=\sup _{0 \neq v \in \mathcal{H}_{Q}} \frac{\left|\langle w, v\rangle_{*}\right|}{\|v\|_{+}}
$$


which satisfies

$$
c^{-1}\|w\|_{-}^{2} \leq\|w\|_{Q, t, *}^{2} \leq c\|w\|_{-}^{2}
$$

for each $t \in[0, T]$ and every $w \in \mathcal{H}_{Q}^{*}$ by virtue of (13).

Next, we consider an evolution system $U_{H}(t, s)_{s, t \in[0, T]}$ on $\mathcal{H}$ consisting of a two-parameter family of linear unitary operators satisfying the usual strong continuity properties and composition laws, as for instance in [21] or [22], along with a class of approximating functions $F_{t}: \mathbb{R}^{+} \mapsto \mathcal{L}(\mathcal{H})$ which satisfy the following hypothesis:

(F) We have $F_{t}(0)=\mathbb{I}$ and there exists a constant $c \in[0,+\infty)$ such that the inequalities

$$
\left\|F_{t}(\tau) v\right\| \leq \exp [c \tau]\|v\|
$$

and

$$
\left\|F_{t}(\tau) v\right\|_{Q, t, *} \leq \exp [c \tau]\|v\|_{Q, t, *}
$$

hold for each $t \in[0, T]$, each $\tau \in \mathbb{R}^{+}$and every $v \in \mathcal{H}$.

For instance, it is plain that both (3) and (4) satisfy these conditions with $c=0$. That is, since $S_{t}(\tau)$ commutes with $H(t)^{-\frac{1}{2}}$ and is unitary on $\mathcal{H}$ we have

$$
\left\|S_{t}(\tau) v\right\|_{Q, t, *}=\|v\|_{Q, t, *}
$$

from (12), so that the inequality

$$
\left\|R_{t}(\tau) v\right\|_{Q, t, *} \leq\|v\|_{Q, t, *}
$$

follows immediately by writing $R_{t}(\tau)$ as the Laplace transform of $S_{t}(\tau)$. However, we remark that in general (18) is not a consequence of (17), nor is (17) a consequence of (18).

Now let $\mathcal{L}\left(\mathcal{H}_{Q}, \mathcal{H}_{Q}^{*}\right)$ be the space of all linear bounded operators from $\mathcal{H}_{Q}$ into $\mathcal{H}_{Q}^{*}$. In order to formulate our requirements regarding $U_{H}(t, s)$, we introduce the unique operator $\mathrm{H}(t) \in \mathcal{L}\left(\mathcal{H}_{Q}, \mathcal{H}_{Q}^{*}\right)$ characterized by the relation

$$
Q(t)[v, w]=\langle\mathrm{H}(t) v, w\rangle_{*}
$$

for every $t \in[0, T]$ and all $v, w \in \mathcal{H}_{Q}$. It is known that for each such $t$ the operator $\mathrm{H}(t)$ is an extension of the self-adjoint generator $H(t)$, and that

$$
\mathcal{D}(H(t))=\left\{v \in \mathcal{H}_{Q}: \mathrm{H}(t) v \in \mathcal{H}\right\}
$$

(see for instance [16]). Our hypothesis concerning $U_{H}(t, s)$ then consists of the following three parts:

(U) We have $U_{H}(t, s)\left(\mathcal{H}_{Q}\right) \subseteq \mathcal{H}_{Q}$ for all $s, t \in[0, T]$ with $t \geq s$, that is, $U_{H}(t, s)$ leaves $\mathcal{H}_{Q}$ invariant. Moreover the following conditions are satisfied: 
(a) For every $v \in \mathcal{H}_{Q}$ the relation

$$
\lim _{\tau \rightarrow 0_{+}} \sup _{t \in[s, T]}\left\|\frac{F_{t}(\tau)-\mathbb{I}}{\tau} U_{H}(t, s) v+i \mathrm{H}(t) U_{H}(t, s) v\right\|_{-}=0
$$

holds.

(b) For all $v, w \in \mathcal{H}_{Q}$ the function $t \mapsto\left\langle U_{H}(t, s) v, w\right\rangle_{*}$ is differentiable on $[0, T]$ and we have

$$
i \frac{d}{d t}\left\langle U_{H}(t, s) v, w\right\rangle_{*}=\left\langle\mathrm{H}(t) U_{H}(t, s) v, w\right\rangle_{*}
$$

for all $s, t \in[0, T]$ with $t>s$.

(c) For every $v \in \mathcal{H}_{Q}$ the function $t \mapsto \mathrm{H}(t) U_{H}(t, s) v$ is continuous on $[0, T]$ in the strong topology of $\mathcal{H}_{Q}^{*}$.

What (20) does is to identify the right-derivative of $\tau \mapsto F_{t}(\tau)$ at the origin with the operator $-i \mathrm{H}(t)$ in the strong topology of $\mathcal{H}_{Q}^{*}$, while (21) is interpreted as the weak form of (1) we alluded to earlier, with $u(t)=U_{H}(t, s) v$.

Under the above conditions our main result is the following.

Theorem. Assume that Hypotheses (Q), (F) and (U) hold. Then for all $s, t \in[0, T]$ with $t \geq s$ we have

$$
U_{H}(t, s)=\lim _{n \rightarrow+\infty} \prod_{\gamma=n-1}^{0} F_{s+\frac{\gamma}{n}(t-s)}\left(\frac{t-s}{n}\right)
$$

in the weak operator topology of $\mathcal{L}(\mathcal{H})$. In particular, if the $F_{t}(\tau)$ 's are also unitary, then (22) holds in the strong operator topology of $\mathcal{L}(\mathcal{H})$.

Remark. Since the $U_{H}(t, s)$ 's are unitary we have

$$
U_{H}(t, s)=U_{H}^{*}(s, t)
$$

for all $s, t \in[0, T]$, where $U_{H}^{*}(s, t)$ denotes the adjoint of $U_{H}(s, t)$ in $\mathcal{L}(\mathcal{H})$. Consequently, from (22) we immediately obtain

$$
U_{H}(t, s)=\lim _{n \rightarrow+\infty} \prod_{\gamma=0}^{n-1} F_{t+\frac{\gamma}{n}(s-t)}^{*}\left(\frac{s-t}{n}\right)
$$

for all $s, t \in[0, T]$ with $t \leq s$ in the weak operator topology of $\mathcal{L}(\mathcal{H})$. Therefore, in the sequel we shall formulate our results only for the case $t \geq s$.

In view of the applications to quantum mechanics, a particularly interesting illustration of this theorem obtains when the operators $H(t)$ are of the form

$$
H(t)=H_{0} \dot{+} V(t)
$$


where $H_{0}$ is a time-independent, self-adjoint, positive operator and $V(t)_{t \in[0, T]}$ a one-parameter family of self-adjoint operators on $\mathcal{H}$, the meaning of (23) being that of a quadratic form sum.

In order to display our result in this case we need to rephrase slightly the hypotheses of Corollary II.28 in [21]. Let $Q_{0}$ be the closed, Hermitian and positive sesquilinear form associated with $H_{0}$ and let $Q_{V}(t)_{t \in[0, T]}$ be the oneparameter family of closed and Hermitian sesquilinear forms associated with the $V(t)^{\prime}$ 's. We assume that $Q_{V}(t)$ is relatively bounded with respect to $Q_{0}$ uniformly in $t$. Writing $\mathcal{H}_{0}:=\mathcal{D}\left(H_{0}^{\frac{1}{2}}\right)$ for the domain of $Q_{0}$, this means that the domain of $Q_{V}(t)$ contains $\mathcal{H}_{0}$ for every $t \in[0, T]$ and that the following hypothesis is valid (see, for instance, [14] for a discussion of this notion):

(V) There exist constants $a \in[0,1)$ and $b \in \mathbb{R}$ such that the inequality

$$
\left|Q_{V}(t)[v, v]\right| \leq a Q_{0}[v, v]+b\|v\|^{2}
$$

holds for each $t \in[0, T]$ and every $v \in \mathcal{H}_{0}$.

In order to realize (23) as a form sum we write

$$
Q(t)=Q_{0}+Q_{V}(t)
$$

for every $t \in[0, T]$. Thus we have $\mathcal{H}_{0}=\mathcal{H}_{Q}$ and the preceding assumptions imply the existence of unique operators $\mathrm{H}(t), \mathrm{H}_{0}, \mathrm{~V}(t) \in \mathcal{L}\left(\mathcal{H}_{Q}, \mathcal{H}_{Q}^{*}\right)$ satisfying (19) and the relations

$$
\begin{gathered}
Q_{0}[v, w]=\left\langle\mathrm{H}_{0} v, w\right\rangle_{*}, \\
Q_{V}(t)[v, w]=\langle\mathrm{V}(t) v, w\rangle_{*}
\end{gathered}
$$

for every $t \in[0, T]$ and all $v, w \in \mathcal{H}_{Q}$, respectively. Consequently (24) reads

$$
\left|\langle\mathrm{V}(t) v, v\rangle_{*}\right| \leq a\left\langle\mathrm{H}_{0} v, v\right\rangle_{*}+b\|v\|^{2}
$$

and the combination of (25), (26) and (27) gives

$$
\mathrm{H}(t)=\mathrm{H}_{0}+\mathrm{V}(t)
$$

as an equality in $\mathcal{L}\left(\mathcal{H}_{Q}, \mathcal{H}_{Q}^{*}\right)$, which is indeed the meaning of (23).

The second relevant hypothesis is the following:

$\left(\mathrm{V}^{\prime}\right)$ The $\mathcal{L}\left(\mathcal{H}_{Q}, \mathcal{H}_{Q}^{*}\right)$-valued function $t \mapsto \mathrm{V}(t)$ is strongly differentiable on $[0, T]$ and its derivative $\mathrm{V}^{\prime}(t) \in \mathcal{L}\left(\mathcal{H}_{Q}, \mathcal{H}_{Q}^{*}\right)$ satisfies

$$
\left|\left\langle\mathrm{V}^{\prime}(t) v, v\right\rangle_{*}\right| \leq a\left\langle\mathrm{H}_{0} v, v\right\rangle_{*}+b\|v\|^{2}
$$

for each $t \in[0, T]$ and every $v \in \mathcal{H}_{Q}$, where $a$ and $b$ are as in (24). 
The implication of Corollary II.28 in [21] is then that there exists a unitary evolution system $U_{H_{0}+V}(t, s)_{s, t \in[0, T]}$ on $\mathcal{H}$ satisfying parts (b) and (c) of $\mathrm{Hy}$ pothesis $(\mathrm{U})$, where $\mathrm{H}(t)$ is given by (28). What we wish to display here are important ways in which we can approximate $U_{H_{0}+V}(t, s)$.

Corollary. Assume that Hypotheses $(V),\left(V^{\prime}\right)$ and $(F)$ hold. Assume furthermore that (20) is valid. Then the conclusion of the theorem holds true for $U_{H_{0}+V}(t, s)$. In particular, for all $s, t \in[0, T]$ with $t \geq s$ we have

$$
U_{H_{0}+V}(t, s)=\lim _{n \rightarrow+\infty} \prod_{\gamma=n-1}^{0}\left(\mathbb{I}+i \frac{t-s}{n} H\left(s+\frac{\gamma}{n}(t-s)\right)\right)^{-1}
$$

in the weak operator topology of $\mathcal{L}(\mathcal{H})$. Moreover, we also have

$$
U_{H_{0}+V}(t, s)=\lim _{n \rightarrow+\infty} \prod_{\gamma=n-1}^{0} \exp \left[-i \frac{t-s}{n} H\left(s+\frac{\gamma}{n}(t-s)\right)\right]
$$

in the strong operator topology of $\mathcal{L}(\mathcal{H})$.

REmarks. (1) The above results can all be modified in a straightforward manner to cover the case where the $H(t)$ 's are self-adjoint operators uniformly bounded from below. Thus, everywhere in the sequel we shall only consider positive generators, a restriction that we will also apply to the two examples of Section 4 .

(2) A relation similar to (31) was derived in Appendix B of [9] in a more specific context and on the basis of a technique different from the one we develop in the next section, which provides a simple and natural framework for the proofs of our general results.

(3) On the right-hand side of (30) and (31) the operator $H(t)$ appears as a whole, although it splits as in (23). A natural question is thus whether formulae such as

$$
\begin{aligned}
& U_{H_{0}+V}(t, s) \\
& =\lim _{n \rightarrow+\infty} \prod_{\gamma=n-1}^{0}\left(\mathbb{I}+i \frac{t-s}{n} H_{0}\right)^{-1}\left(\mathbb{I}+i \frac{t-s}{n} V\left(s+\frac{\gamma}{n}(t-s)\right)\right)^{-1}
\end{aligned}
$$

and

$$
\begin{aligned}
& U_{H_{0}+V}(t, s) \\
& =\lim _{n \rightarrow+\infty} \prod_{\gamma=n-1}^{0} \exp \left[-i \frac{t-s}{n} H_{0}\right] \exp \left[-i \frac{t-s}{n} V\left(s+\frac{\gamma}{n}(t-s)\right)\right]
\end{aligned}
$$

are also true under our general conditions. It turns out that this problem remains open, although there have been numerous extensions of Trotter's original 
work [23] over the years concerning the linear autonomous case, including those appearing in [3]-[5], [10], [12], [13] and [17] (see, for instance, [7] for a comprehensive analysis of some of these works). The linear non-autonomous case is more difficult, unless the domains of the $H(t)$ 's are independent of time and the evolution equations satisfied in a classical sense, as in [11].

We shall dwell a bit more on this at the end of the next section, by pointing out where the difficulties are.

\section{Proof of the Results}

In what follows we write $c$ for all the irrelevant constants that occur in the various estimates unless we specify these constants otherwise. We first draw an elementary but important consequence from the Lipschitz continuity estimate (14).

Lemma 1. Assume that Hypothesis (Q) is valid. Then there exists a constant $c \in(0,+\infty)$ such that the inequality

$$
\|w\|_{Q, t, *} \leq \exp [c|t-s|]\|w\|_{Q, s, *}
$$

holds for all $s, t \in[0, T]$ and every $w \in \mathcal{H}_{Q}^{*}$.

Proof. From (13) and (14) we have

$$
\|v\|_{Q, t}^{2}=Q(t)[v, v] \leq(1+c|t-s|) Q(s)[v, v] \leq \exp [c|t-s|]\|v\|_{Q, s}^{2}
$$

for every $v \in \mathcal{H}_{Q}$, and by symmetry

$$
\|v\|_{Q, s}^{2} \leq \exp [c|t-s|]\|v\|_{Q, t}^{2} .
$$

Therefore we obtain

$$
\sup _{0 \neq v \in \mathcal{H}_{Q}} \frac{\left|\langle w, v\rangle_{Q, *}\right|}{\|v\|_{Q, t}} \leq \exp [c|t-s|] \sup _{0 \neq v \in \mathcal{H}_{Q}} \frac{\left|\langle w, v\rangle_{Q, *}\right|}{\|v\|_{Q, s}},
$$

which is (34) by changing the value of $c$ if necessary.

Without restricting the generality we now assume that $s<t<T$ and set $h=\frac{t-s}{n}$ for $n$ sufficiently large. The preceding lemma then allows us to prove the following result.

Lemma 2. Assume that Hypothesis (Q) and (18) are valid. Then there exists a constant $c \in(0,+\infty)$ such that the estimate

$$
\left\|\prod_{\alpha=n}^{\gamma+1} F_{s+(\alpha-1) h}(h) v\right\|_{-} \leq c\|v\|_{-}
$$


holds for each $\gamma \in\{1, \ldots, n-1\}$ and every $v \in \mathcal{H}$.

Proof. According to (16) this is equivalent to proving that

$$
\left\|\prod_{\alpha=n}^{\gamma+1} F_{s+(\alpha-1) h}(h) v\right\|_{Q, t, *} \leq c\|v\|_{Q, t, *} .
$$

For this we apply (34) and (18) alternatingly. After $2(n-\gamma)-1$ steps we obtain

$$
\begin{aligned}
& \left\|\prod_{\alpha=n}^{\gamma+1} F_{s+(\alpha-1) h}(h) v\right\|_{Q, t, *} \\
& \leq \exp [c(2(n-\gamma)-1) h]\left\|F_{t-(n-\gamma) h}(h) v\right\|_{Q, t-(n-\gamma) h, *}
\end{aligned}
$$

since $n h=t-s$. Furthermore, we can estimate the last factor in (36) as

$$
\begin{aligned}
& \left\|F_{t-(n-\gamma) h}(h) v\right\|_{Q, t-(n-\gamma) h, *} \\
& \leq \exp [c h]\|v\|_{Q, t-(n-\gamma) h, *} \leq \exp [c(n-\gamma+1) h]\|v\|_{Q, t, *}
\end{aligned}
$$

by first applying (18) and then (34). Consequently, the substitution of (37) into (36) leads to the inequality

$$
\begin{aligned}
& \left\|\prod_{\alpha=n}^{\gamma+1} F_{s+(\alpha-1) h}(h) v\right\|_{Q, t, *} \\
& \leq \exp [3 c(n-\gamma) h]\|v\|_{Q, t, *} \leq \exp [3 c n h]\|v\|_{Q, t, *},
\end{aligned}
$$

which gives the desired result since $n h=t-s \leq T$.

We now define the sequence $\left(P_{n}(t, s)\right) \subset \mathcal{L}(\mathcal{H})$ by

$$
P_{n}(t, s):=U_{H}(t, s)-\prod_{\gamma=n}^{1} F_{s+(\gamma-1) h}(h)
$$

and establish the following useful preliminary estimate for it.

Lemma 3. Assume that Hypothesis (Q) and (18) hold. Then we have the inequality

$$
\begin{aligned}
& \left\|P_{n}(t, s) v\right\|_{-} \\
& \leq c n \sup _{r \in[s, t]}\left\|U_{H}(r+h, s) v-F_{r}(h) U_{H}(r, s) v\right\|_{-}
\end{aligned}
$$

for every $v \in \mathcal{H}$. 
Proof. From the basic composition laws for the $U_{H}(t, s)$ 's, $(38)$, and remembering that $t=s+n h$ we first get

$$
\begin{aligned}
& P_{n}(t, s) \\
& =\prod_{\gamma=n}^{1} U_{H}(s+\gamma h, s+(\gamma-1) h)-\prod_{\gamma=n}^{1} F_{s+(\gamma-1) h}(h) \\
& =\prod_{\alpha=n}^{2} F_{s+(\alpha-1) h}(h) \times\left(U_{H}(s+h, s)-F_{s}(h)\right) \\
& +\sum_{\gamma=2}^{n-1} \prod_{\alpha=n}^{\gamma+1} F_{s+(\alpha-1) h}(h) \times\left(U_{H}(s+\gamma h, s+(\gamma-1) h)-F_{s+(\gamma-1) h}(h)\right) \\
& \times \prod_{\beta=\gamma-1}^{1} U_{H}(s+\beta h, s+(\beta-1) h) \\
& +\left(U_{H}(t, t-h)-F_{t-h}(h)\right) \times \prod_{\beta=n-1}^{1} U_{H}(s+\beta h, s+(\beta-1) h)
\end{aligned}
$$

where the second equality follows from the cancellation of all but the two relevant terms in the expression on its right-hand side. Furthermore, by repeated applications of the composition laws we have

$$
\prod_{\beta=\gamma-1}^{1} U_{H}(s+\beta h, s+(\beta-1) h)=U_{H}(s+(\gamma-1) h, s)
$$

and

$$
\prod_{\beta=n-1}^{1} U_{H}(s+\beta h, s+(\beta-1) h)=U_{H}(t-h, s)
$$

for the two products that appear on the right-hand side of (40). Substituting (41) and (42) into (40), multiplying out and regrouping terms we then get

$P_{n}(t, s) v$

$=\sum_{\gamma=1}^{n-1} \prod_{\alpha=n}^{\gamma+1} F_{s+(\alpha-1) h}(h) \times\left(U_{H}(s+\gamma h, s)-F_{s+(\gamma-1) h}(h) U_{H}(s+(\gamma-1) h, s)\right) v$

$+\left(U_{H}(t, s)-F_{t-h}(h) U_{H}(t-h, s)\right) v$

for every $v \in \mathcal{H}$ since $U_{H}(s, s)=\mathbb{I}$.

We now proceed by estimating the norm of the first term on the right-hand 
side of (43) by means of (35); we obtain

$$
\begin{aligned}
& \sum_{\gamma=1}^{n-1}\left\|\prod_{\alpha=n}^{\gamma+1} F_{s+(\alpha-1) h}(h) \times\left(U_{H}(s+\gamma h, s)-F_{s+(\gamma-1) h}(h) U_{H}(s+(\gamma-1) h, s)\right) v\right\|_{-} \\
& \quad \leq c \sum_{\gamma=1}^{n-1}\left\|U_{H}(s+\gamma h, s) v-F_{s+(\gamma-1) h}(h) U_{H}(s+(\gamma-1) h, s) v\right\|_{-}
\end{aligned}
$$

so that the combination of (43) and (44) gives

$$
\begin{aligned}
& \left\|P_{n}(t, s) v\right\|_{-} \\
& \leq c \sum_{\gamma=1}^{n}\left\|U_{H}(s+\gamma h, s) v-F_{s+(\gamma-1) h}(h) U_{H}(s+(\gamma-1) h, s) v\right\|_{-} .
\end{aligned}
$$

If we now set $r_{\gamma}:=s+(\gamma-1) h$ we get a fortiori

$$
\begin{aligned}
& \left\|P_{n}(t, s) v\right\|_{-} \\
& \leq c n \max _{\gamma \in\{1, \ldots, n\}}\left\|U_{H}\left(r_{\gamma}+h, s\right) v-F_{r_{\gamma}}(h) U_{H}\left(r_{\gamma}, s\right) v\right\|_{-} \\
& \leq c n \sup _{r \in[s, t-h]}\left\|U_{H}(r+h, s) v-F_{r}(h) U_{H}(r, s) v\right\|_{-},
\end{aligned}
$$

which indeed leads to $(39)$.

In order to estimate (39) further we now introduce two linear operators defined on $\mathcal{H}_{Q}$, namely,

$$
L(h, r):=h^{-1}\left(\mathbb{I}-F_{r}(h)\right)-i \mathrm{H}(r)
$$

and

$$
M(h, r):=h^{-1}\left(\mathbb{I}-U_{H}(r+h, r)\right)-i \mathrm{H}(r)
$$

where $\mathrm{H}(r)$ stands for the operator defined by (19). We can then express the right-hand side of (39) somewhat differently as in the following result, albeit now with the additional but harmless restriction $v \in \mathcal{H}_{Q}$.

Lemma 4. Assume that Hypothesis (Q) and (18) hold, along with the invariance part of Hypothesis $(U)$. Then we have the inequality

$$
\begin{aligned}
& \left\|P_{n}(t, s) v\right\|_{-} \\
& \leq c \sup _{r \in[s, t]}\left\|L(h, r) U_{H}(r, s) v-M(h, r) U_{H}(r, s) v\right\|_{-}
\end{aligned}
$$

for every $v \in \mathcal{H}_{Q}$.

Proof. From (45) and (46) we obtain

$$
\begin{aligned}
& h L(h, r) U_{H}(r, s) v \\
& \quad=U_{H}(r, s) v-F_{r}(h) U_{H}(r, s) v-i h \mathrm{H}(r) U_{H}(r, s) v
\end{aligned}
$$


and

$$
\begin{aligned}
& h M(h, r) U_{H}(r, s) v \\
& \quad=U_{H}(r, s) v-U_{H}(r+h, s) v-i h \mathrm{H}(r) U_{H}(r, s) v,
\end{aligned}
$$

respectively, where we have used the composition laws to establish (49). By subtracting (49) from (48) we then get

$$
\begin{aligned}
& h L(h, r) U_{H}(r, s) v-h M(h, r) U_{H}(r, s) v \\
& =U_{H}(r+h, s) v-F_{r}(h) U_{H}(r, s) v
\end{aligned}
$$

so that (47) indeed follows from (39) since $n h=t-s \leq T$.

We are now ready for the following.

Proof of the theorem. We first show that

$$
\lim _{n \rightarrow+\infty}\left\|P_{n}(t, s) v\right\|_{-}=0
$$

for every $v \in \mathcal{H}_{Q}$. For this it is sufficient to have

$$
\lim _{n \rightarrow+\infty} \sup _{r \in[s, t]}\left\|L(h, r) U_{H}(r, s) v\right\|_{-}=0
$$

and

$$
\lim _{n \rightarrow+\infty} \sup _{r \in[s, t]}\left\|M(h, r) U_{H}(r, s) v\right\|_{-}=0
$$

according to (47). Referring back to (48) we see that (51) is equivalent to having

$$
\lim _{n \rightarrow+\infty} \sup _{r \in[s, t]}\left\|h^{-1}\left(U_{H}(r, s) v-F_{r}(h) U_{H}(r, s) v\right)-i \mathrm{H}(r) U_{H}(r, s) v\right\|_{-}=0
$$

for every $v \in \mathcal{H}_{Q}$, which is an immediate consequence of (20).

As for the proof of (52) we start from the relation

$$
\begin{aligned}
& \left\langle M(h, r) U_{H}(r, s) v, w\right\rangle_{*} \\
& =i h^{-1} \int_{r}^{r+h} d k\left\langle\mathrm{H}(k) U_{H}(k, s) v-\mathrm{H}(r) U_{H}(r, s) v, w\right\rangle_{*}
\end{aligned}
$$

valid for every $w \in \mathcal{H}_{Q}$, which follows from (21) and (49). Relation (53) then leads to

$$
\begin{aligned}
& \left\|M(h, r) U_{H}(r, s) v\right\|_{-} \\
& \leq h^{-1} \int_{r}^{r+h} d k\left\|\mathrm{H}(k) U_{H}(k, s) v-\mathrm{H}(r) U_{H}(r, s) v\right\|_{-},
\end{aligned}
$$

and since the function $k \mapsto \mathbf{H}(k) U_{H}(k, s) v$ is uniformly continuous on $[r, r+h]$ with respect to the strong topology of $\mathcal{H}_{Q}^{*}$ according to (c) of Hypothesis (U), 
we conclude that for every $\epsilon \in(0,+\infty)$ there exists $h_{\epsilon} \in(0,+\infty)$ such that the inequalities $0 \leq k-r \leq h \leq h_{\epsilon}$ along with (54) imply the estimate

$$
\sup _{r \in[s, t]}\left\|M(h, r) U_{H}(r, s) v\right\|_{-} \leq \epsilon,
$$

which is equivalent to (52). Consequently (50) holds, which implies that

$$
\left(U_{H}(t, s) v, w\right)=\lim _{n \rightarrow+\infty}\left(\prod_{\gamma=n-1}^{0} F_{s+\frac{\gamma}{n}(t-s)}\left(\frac{t-s}{n}\right) v, w\right)
$$

for all $v, w \in \mathcal{H}_{Q}$ according to (15) and (38), since $\langle., .\rangle_{*}$ and (.,.) are interchangeable on $\mathcal{H}$.

In order to prove (22), it thus remains to extend (55) to all $v, w \in \mathcal{H}$. On the one hand, as a vector subspace $\mathcal{H}_{Q}$ is dense in $\mathcal{H}$ relative to the strong topology of this latter space. On the other hand, arguing as in the proof of Lemma 2 we infer from (17) that the estimate

$$
\left\|\prod_{\gamma=n-1}^{0} F_{s+\frac{\gamma}{n}(t-s)}\left(\frac{t-s}{n}\right) v\right\| \leq c\|v\|
$$

holds for every $v \in \mathcal{H}$ for some $c \in(0,+\infty)$ independent of $n$. Therefore, the fact that (55) holds for all $v, w \in \mathcal{H}$ follows from a standard density argument.

The very last statement of the theorem is obvious since the weak and strong topologies of $\mathcal{H}$ coincide on the unitary group in $\mathcal{L}(\mathcal{H})$.

We now turn to the proof of the corollary, which first requires the verification of Hypothesis (Q).

Lemma 5. Assume that Hypotheses $(V)$ and $\left(V^{\prime}\right)$ are valid. Then relations (13) and (14) hold relative to the fixed norm

$$
\|v\|_{+}:=\left\|H_{0}^{\frac{1}{2}} v\right\|
$$

on $\mathcal{H}_{Q}$.

Proof. From (24), (25) and (56) we get

$$
(1-a)\|v\|_{+}^{2}-b\|v\|^{2} \leq Q(t)[v, v] \leq(1+a)\|v\|_{+}^{2}+b\|v\|^{2}
$$

for each $t \in[0, T]$ and every $v \in \mathcal{H}_{Q}$, which leads to (13) by virtue of (7) and the first embedding in (11).

The starting point for the proof of (14) is the relation

$$
Q(t)[v, v]-Q(s)[v, v]=\int_{s}^{t} d \tau\left\langle\mathrm{V}^{\prime}(\tau) v, v\right\rangle_{*}
$$


which follows from $(25),(27)$ and the differentiability of $\mathrm{V}$. We then obtain the desired estimate

$$
\begin{aligned}
& |Q(t)[v, v]-Q(s)[v, v]| \\
& \leq \int_{s}^{t} d \tau\left|\left\langle\mathrm{V}^{\prime}(\tau) v, v\right\rangle_{*}\right| \leq c^{*}|t-s|\|v\|_{+}^{2}
\end{aligned}
$$

for all $s, t \in[0, T]$ and every $v \in \mathcal{H}_{Q}$, as a consequence of (29), (56) and the first embedding in (11) once again.

REMARK. It is also possible to obtain the first inequality in (13) from (24) and the condition

$$
Q_{0}[v, v] \geq \kappa\|v\|^{2}
$$

for a sufficiently large positive $\kappa$, instead of invoking (7). This is particularly useful when (7) cannot easily be proved directly, as will be the case in the second example of Section 4.

Since we know from Corollary II.28 and its proof in [21] that $U_{H_{0}+V}(t, s)$ satisfies parts (b) and (c) of Hypothesis (U), the preceding lemma and the theorem imply the first statement of the corollary. Moreover, we have already noted that the resolvent operators (3) and the unitary semigroup (4) satisfy Hypothesis (F) in a trivial way. Therefore, in order to prove (30) and (31) it remains to verify part (a) of Hypothesis (U) for (3) and (4). For this it is necessary to consider the $\mathcal{C}_{0}$-semigroup on $\mathcal{H}_{Q}^{*}$ given by

$$
\mathrm{S}_{t}(\tau):=\exp [-i \tau \mathrm{H}(t)],
$$

namely, the extension by continuity of (4) to the whole of $\mathcal{H}_{Q}^{*}$. It is easily verified that (58) is unitary with respect to the norm (12), and that its infinitesimal generator is indeed $-i \mathrm{H}(t)$, considered this time as an unbounded operator in $\mathcal{H}_{Q}^{*}$ defined on the dense subspace $\mathcal{H}_{Q}$ where $\mathrm{H}(t)$ is self-adjoint.

We begin with the following intermediary result, valid quite generally and independently of (28).

Lemma 6. Assume that (13) of Hypothesis (Q) holds. Then we have

$$
\lim _{\tau \rightarrow 0_{+}} \sup _{t \in[0, T]}\|(\exp [-i \tau \mathbf{H}(t)]-\mathbb{I}) v\|_{-}=0
$$

for every $v \in \mathcal{H}_{Q}^{*}$. Moreover, for any compact set $\mathcal{K} \subset \mathcal{H}_{Q}^{*}$ the limit (59) is uniform in $v \in \mathcal{K}$.

Proof. Relation (13) implies (16). Consequently, from the properties of $\exp [-i \tau \mathrm{H}(t)]$ and from the fact that $\mathcal{H}_{Q}$ is dense in $\mathcal{H}_{Q}^{*}$ as a vector subspace, it is sufficient to prove the relation

$$
\lim _{\tau \rightarrow 0_{+}} \sup _{t \in[0, T]}\|(\exp [-i \tau H(t)]-\mathbb{I}) v\|_{-}=0
$$


for every $v \in \mathcal{H}_{Q}$. We first show that the identity

$$
\begin{aligned}
& \langle\exp [-i \tau H(t)] v-v, w\rangle_{*} \\
& =-i \int_{0}^{\tau} d \sigma\left(\exp [-i \sigma H(t)] H^{\frac{1}{2}}(t) v, H^{\frac{1}{2}}(t) w\right)
\end{aligned}
$$

holds for each $\tau \in[0,+\infty)$, every $t \in[0, T]$ and all $v, w \in \mathcal{H}_{Q}$.

Indeed, from a classic property of $\mathcal{C}_{0}$-semigroups we may write

$$
\langle\exp [-i \tau H(t)] v-v, w\rangle_{*}=-i \int_{0}^{\tau} d \sigma(\exp [-i \sigma H(t)] H(t) v, w)
$$

for each $v \in \mathcal{D}(H(t))$ and every $w \in \mathcal{H}_{Q}$. But $v \in \mathcal{D}(H(t))$ if, and only if, $H^{\frac{1}{2}}(t) v \in \mathcal{H}_{Q}$; furthermore $H^{\frac{1}{2}}(t)$ commutes with $\exp [-i \sigma H(t)]$ on $\mathcal{H}_{Q}$ and is self-adjoint in $\mathcal{H}$, so that (61) holds for each $\tau \in[0,+\infty)$ and all $t \in[0, T]$, $v \in \mathcal{D}(H(t)), w \in \mathcal{H}_{Q}$.

Therefore, in order to show the validity of (61) for all $v \in \mathcal{H}_{Q}$ it suffices to prove that $\mathcal{D}(H(t))$ is dense in $\mathcal{H}_{Q}$. On the one hand, the restriction of $\exp [-i \tau H(t)]$ to $\mathcal{H}_{Q}$ defines a $\mathcal{C}_{0}$-semigroup there, the generator of which being consequently densely defined in $\mathcal{H}_{Q}$. On the other hand, the domain of that generator is contained in $\mathcal{D}(H(t))$ by virtue of the first embedding in (11). We can then conclude that $\mathcal{D}(H(t))$ is a fortiori dense in $\mathcal{H}_{Q}$, so that (61) holds for all $v \in \mathcal{H}_{Q}$.

It is now easy to derive (60) from (61), since Schwarz inequality and the fact that $\exp [-i \tau H(t)]$ is unitary in $\mathcal{H}$ lead to the estimate

$$
\begin{aligned}
& \left|\langle\exp [-i \tau H(t)] v-v, w\rangle_{*}\right| \\
& \leq \tau\left\|H^{\frac{1}{2}}(t) v\right\|\left\|H^{\frac{1}{2}}(t) w\right\| \leq c \tau\|v\|_{+}\|w\|_{+}
\end{aligned}
$$

as a consequence of (9) and (13), where $c$ is independent of $t$. Thus we get

$$
\sup _{t \in[0, T]} \sup _{0 \neq w \in \mathcal{H}_{Q}} \frac{\left|\langle\exp [-i \tau H(t)] v-v, w\rangle_{*}\right|}{\|w\|_{+}} \leq c \tau\|v\|_{+} \rightarrow 0
$$

as $\tau \rightarrow 0_{+}$, which is the desired result.

As for the very last assertion of the lemma, we remark that the operator norm of $\exp [-i \tau \mathrm{H}(t)]-\mathbb{I}$ in $\mathcal{L}\left(\mathcal{H}_{Q}^{*}\right)$ satisfies

$$
\sup _{\tau \in[0,+\infty)} \sup _{t \in[0, T]}\|\exp [-i \tau \mathrm{H}(t)]-\mathbb{I}\|_{\mathcal{L}\left(\mathcal{H}_{Q}^{*}\right)}<+\infty
$$

so that the statement follows for instance from Lemma 3 in [24].

The preceding considerations now allow us to prove the desired assertions.

Proof of the Corollary. As already observed it remains to verify part (a) of Hypothesis (U) for the approximating functions (3) and (4). Since the former 
is the Laplace transform of the latter, we begin with (4). This means that we must have

$$
\lim _{\tau \rightarrow 0_{+}} \sup _{t \in[0, T]}\left\|\frac{\exp [-i \tau H(t)]-\mathbb{I}}{\tau} U_{H_{0}+V}(t, s) v+i \mathrm{H}(t) U_{H_{0}+V}(t, s) v\right\|_{-}=0
$$

for each $v \in \mathcal{H}_{Q}$ and every $s \in[0, T]$, where $\mathrm{H}(t)$ is given by (28).

Remembering that $\exp [-i \tau H(t)]$ and $\exp [-i \tau \mathrm{H}(t)]$ coincide on $\mathcal{H}_{Q}$ we may write

$$
\begin{aligned}
& \frac{\exp [-i \tau H(t)]-\mathbb{I}}{\tau} U_{H_{0}+V}(t, s) v+i \mathrm{H}(t) U_{H_{0}+V}(t, s) v \\
& =-\frac{i}{\tau} \int_{0}^{\tau} d \sigma(\exp [-i \sigma \mathrm{H}(t)]-\mathbb{I}) \mathrm{H}(t) U_{H_{0}+V}(t, s) v
\end{aligned}
$$

for each $\tau \in(0,+\infty)$ and every $v \in \mathcal{H}_{Q}$, since $-i \mathrm{H}(t)$ is the infinitesimal generator of $\exp [-i \tau \mathrm{H}(t)]$ in $\mathcal{H}_{Q}^{*}$, and since the invariance property of Hypothesis (U) holds in this case. Therefore we obtain

$$
\begin{aligned}
& \left\|\frac{\exp [-i \tau H(t)]-\mathbb{I}}{\tau} U_{H_{0}+V}(t, s) v+i \mathrm{H}(t) U_{H_{0}+V}(t, s) v\right\|_{-} \\
& \leq \sup _{\sigma \in[0, \tau]}\left\|(\exp [-i \sigma \mathrm{H}(t)]-\mathbb{I}) \mathrm{H}(t) U_{H_{0}+V}(t, s) v\right\|_{-} .
\end{aligned}
$$

Furthermore, from the proof of Corollary II.28 of [21] we already know that the function $t \mapsto \mathrm{H}(t) U_{H_{0}+V}(t, s) v$ is continuous on $[0, T]$ in the strong topology of $\mathcal{H}_{Q}^{*}$ for each $v \in \mathcal{H}_{Q}$, so that the set

$$
\mathcal{K}:=\left\{w \in \mathcal{H}_{Q}^{*}: w=\mathbf{H}(t) U_{H_{0}+V}(t, s) v, \quad t \in[0, T]\right\}
$$

is compact in $\mathcal{H}_{Q}^{*}$. Relation (63) then follows from (65) and the very last statement of Lemma 6.

The proof of the analogous property for (3) follows from (63) through a Laplace transform argument and dominated convergence. Indeed we have

$$
(\mathbb{I}+i \tau H(t))^{-1} v=\int_{0}^{+\infty} d \sigma \exp [-\sigma] \exp [-i \sigma \tau H(t)] v
$$

for each $\tau \in(0,+\infty)$ and every $v \in \mathcal{H}$ as an improper $\mathcal{H}$-valued Riemann integral, so that

$$
\begin{aligned}
& \frac{(\mathbb{I}+i \tau H(t))^{-1}-\mathbb{I}}{\tau} U_{H_{0}+V}(t, s) v+i \mathrm{H}(t) U_{H_{0}+V}(t, s) v \\
& =\int_{0}^{+\infty} d \sigma \exp [-\sigma] \sigma\left(\frac{\exp [-i \sigma \tau H(t)]-\mathbb{I}}{\sigma \tau} U_{H_{0}+V}(t, s) v+i \mathrm{H}(t) U_{H_{0}+V}(t, s) v\right)
\end{aligned}
$$

since

$$
\int_{0}^{+\infty} d \sigma \exp [-\sigma]=\int_{0}^{+\infty} d \sigma \exp [-\sigma] \sigma=1
$$


Consequently we get

$$
\begin{aligned}
& \sup _{t \in[0, T]}\left\|\frac{(\mathbb{I}+i \tau H(t))^{-1}-\mathbb{I}}{\tau} U_{H_{0}+V}(t, s) v+i \mathrm{H}(t) U_{H_{0}+V}(t, s) v\right\|_{-} \\
& \leq \int_{0}^{+\infty} d \sigma \exp [-\sigma] \sigma A(\sigma, \tau)
\end{aligned}
$$

where we have introduced the auxiliary function

$\sigma \mapsto A(\sigma, \tau):=\sup _{t \in[0, T]}\left\|\frac{\exp [-i \sigma \tau H(t)]-\mathbb{I}}{\sigma \tau} U_{H_{0}+V}(t, s) v+i \mathrm{H}(t) U_{H_{0}+V}(t, s) v\right\|_{-}$,

and for any fixed $\sigma \in(0,+\infty)$ we have

$$
\lim _{\tau \rightarrow 0_{+}} A(\sigma, \tau)=0
$$

by virtue of (63). Now, by using once again (62) and (64) we obtain from (67) the estimate

$$
\begin{aligned}
& A(\sigma, \tau) \\
& \leq \frac{1}{\sigma \tau} \sup _{t \in[0, T]} \int_{0}^{\sigma \tau} d \rho\left\|(\exp [-i \rho \mathrm{H}(t)]-\mathbb{I}) \mathrm{H}(t) U_{H_{0}+V}(t, s) v\right\|_{-} \\
& \leq c \sup _{t \in[0, T]}\left\|\mathrm{H}(t) U_{H_{0}+V}(t, s) v\right\|_{-}<+\infty
\end{aligned}
$$

uniformly in $\sigma$ and $\tau$. But any finite constant is integrable on $(0,+\infty)$ with respect to the measure $d \sigma \exp [-\sigma] \sigma$, so that by dominated convergence relative to this measure along with (66) and (68) we have

$$
\lim _{\tau \rightarrow 0_{+}} \sup _{t \in[0, T]}\left\|\frac{(\mathbb{I}+i \tau H(t))^{-1}-\mathbb{I}}{\tau} U_{H_{0}+V}(t, s) v+i \mathrm{H}(t) U_{H_{0}+V}(t, s) v\right\|_{-}=0
$$

for each $v \in \mathcal{H}_{Q}$ and every $s \in[0, T]$, as desired.

REMARK. Whereas the methods of this article are relevant to prove (30) and (31) where the operator $H(t)$ appears as a whole, they are not quite appropriate to derive formulae such as (32) and (33). Indeed, the natural choice of approximating functions in this case is

$$
F_{t}(\tau)=\left(\mathbb{I}+i \tau H_{0}\right)^{-1}(\mathbb{I}+i \tau V(t))^{-1}
$$

and

$$
F_{t}(\tau)=\exp \left[-i \tau H_{0}\right] \exp [-i \tau V(t)],
$$

respectively. In either case the problem then lies in the verification of Hypothesis (F): whereas (17) trivially holds for both (69) and (70) with $c=0$, (18) can seldom be valid. For instance, in the case of (70) and by virtue of (16) with

$$
\|w\|_{-}:=\left\|H_{0}^{-\frac{1}{2}} w\right\|
$$


we have successively

$$
\left\|F_{t}(\tau) v\right\|_{Q, t, *}^{2} \leq c\left\|H_{0}^{-\frac{1}{2}} \exp [-i \tau V(t)] v\right\|^{2} \leq c^{2}\|\exp [-i \tau V(t)] v\|_{Q, t, *}^{2}
$$

since $H_{0}^{-\frac{1}{2}}$ commutes with the unitary semigroup $\exp \left[-i \tau H_{0}\right]$ on $\mathcal{H}$. Consequently, even under the most favorable hypotheses regarding $V(t)$ we end up getting an estimate of the form

$$
\left\|F_{t}(\tau) v\right\|_{Q, t, *} \leq c \exp [c \tau]\|v\|_{Q, t, *}
$$

with $c \in[1,+\infty)$, instead of (18). But then, it is impossible to derive the crucial uniform estimate (35) since the number of factors in that product depends explicitly on $n$.

We devote the last section to the illustration of our results.

\section{Two Examples}

In what follows we use the standard notations for the usual spaces of Lebesgue integrable functions and for the corresponding Sobolev spaces of functions defined on Euclidean space (see, for instance, [1]). All the functions are complexvalued unless stated otherwise.

ExAmple 1. We consider the initial-value problem in one space dimension

$$
\begin{aligned}
i \frac{\partial u(x, t)}{\partial t} & =\left(-\frac{1}{2} \frac{\partial}{\partial x} \frac{1}{m(x)} \frac{\partial}{\partial x}+V(x)+\sum_{k=1}^{N} \mathrm{~s}_{k}(t) \delta_{x_{k}}\right) u(x, t), \quad(x, t) \in \mathbb{R} \times(s, T] \\
u(x, s) & =v(x), \quad x \in \mathbb{R}
\end{aligned}
$$

corresponding to a particle with variable mass $m$ moving in a potential $V$ perturbed by time-dependent point interactions supported by a discrete set $\left\{x_{1}, \ldots, x_{N}\right\}$, where $N \in \mathbb{N}^{+}$is fixed and arbitrary (see, for instance, [8] and its references for a physical interpretation of related models).

In this case we view (71) as an evolution problem of the form (1) in $\mathcal{H}=$ $L^{2}(\mathbb{R})$, with the operator $H(t)$ formally given by

$$
H(t):=-\frac{1}{2} \frac{\partial}{\partial x} \frac{1}{m(x)} \frac{\partial}{\partial x}+V(x)+\sum_{k=1}^{N} \mathrm{~s}_{k}(t) \delta_{x_{k}} .
$$

Furthermore we impose the following hypotheses:

(MV) We have $0<\frac{1}{m}+m \in L^{\infty}(\mathbb{R})$ and $0 \leq V \in L^{\infty}(\mathbb{R})$

(S) The strengths of the point interactions $\mathrm{s}_{k}:[0, T] \mapsto[0,+\infty)$ are positive and Lipschitz continuous for every $k \in\{1, \ldots, N\}$. 
Under these conditions there exists a self-adjoint realization of $(72)$ in $L^{2}(\mathbb{R})$ as a positive operator on some time-dependent domain $\mathcal{D}(H(t))$, corresponding to the closed and Hermitian sesquilinear form

$$
\begin{aligned}
& Q(t)[v, w] \\
& =\int_{\mathbb{R}} d x\left(\frac{1}{2 m(x)} v^{\prime}(x) \bar{w}^{\prime}(x)+V(x) v(x) \bar{w}(x)\right)+\sum_{k=1}^{N} \mathrm{~s}_{k}(t) v\left(x_{k}\right) \bar{w}\left(x_{k}\right)
\end{aligned}
$$

defined for all $v, w \in \mathcal{D}\left(H(t)^{\frac{1}{2}}\right)=W^{1,2}(\mathbb{R})$ (see [2] for a variety of constructions of this kind, based on von Neumann's theory of self-adjoint extensions for symmetric operators); furthermore inequality (7) holds. We then have the following result.

Proposition 1. Assume that Hypotheses (MV), (S), (F) and (20) are valid. Then there exists a unique unitary evolution system $U_{H}(t, s)_{s, t \in[0, T]}$ on $L^{2}(\mathbb{R})$ associated with the above realization of (72), for which the conclusion of the theorem holds true. In particular, $U_{H}(t, s)$ can be approximated as in (30) and (31) of the corollary.

Proof. For the fixed norm on $\mathcal{H}_{Q}=W^{1,2}(\mathbb{R})$ we choose

$$
\|v\|_{+}=\left(\int_{\mathbb{R}} d x\left|v^{\prime}(x)\right|^{2}\right)^{\frac{1}{2}} .
$$

Conditions (MV) and (S) together with standard one-dimensional Sobolev theory then imply that Hypothesis (Q) holds. Moreover, (MV) and (S) also guarantee the existence of a unique unitary evolution system $U_{H}(t, s)_{s, t \in[0, T]}$ on $L^{2}(\mathbb{R})$, which leaves $W^{1,2}(\mathbb{R})$ invariant and satisfies parts (b) and (c) of Hypothesis (U) according to Theorem 6.1 in [18] and its proof. Since (F) and (20) are assumed to hold, the conclusion of the theorem follows in this case. The proofs of (30) and (31) are identical to those given at the very end of Section 3.

Example 2. We now consider the initial-value problem

$$
\begin{aligned}
i \frac{\partial u(x, t)}{\partial t} & =\left(-\triangle_{x}+\kappa+V(x, t)\right) u(x, t), \quad(x, t) \in \mathbb{R}^{3} \times(s, T], \\
u(x, s) & =v(x), \quad x \in \mathbb{R}^{3},
\end{aligned}
$$

describing the motion of a quantum particle with constant mass in $\mathbb{R}^{3}$, subjected to a time-dependent potential $V$, measurable in $(x, t)$ and satisfying Rollnik's condition

$$
\int_{\mathbb{R}^{3} \times \mathbb{R}^{3}} d x d y \frac{|V(x, t)||V(y, t)|}{|x-y|^{2}}<+\infty
$$

for every $t \in[0, T]$ (see [21] for a systematic analysis of Rollnik potentials and the rôle of these in quantum mechanics). Here we consider (74) as an evolution problem of the form (1) in $\mathcal{H}=L^{2}\left(\mathbb{R}^{3}\right)$, with

$$
H(t):=-\triangle_{x}+\kappa+V(x, t)
$$


realized as a self-adjoint operator on some time-dependent domain $\mathcal{D}(H(t))$. As is well-known, this is made possible by an application of the Kato-Rellich theorem for forms, provided we define $H_{0}:=-\triangle_{x}+\kappa$ as the self-adjoint, positive operator on the domain

$$
\mathcal{D}\left(H_{0}\right)=W^{2,2}\left(\mathbb{R}^{3}\right),
$$

in which case we have $\mathcal{H}_{Q}=W^{1,2}\left(\mathbb{R}^{3}\right)$ (see, for instance, [14] or [21]). Here we choose $\kappa$ positive and sufficiently large, in relation to our remark immediately following the proof of Lemma 5 in Section 3.

In order to illustrate our theory with this example we need additional requirements on $V$ that ensure some kind of uniformity in $t$. For instance, we can impose the following two hypotheses:

(R) We have

$$
\int_{\mathbb{R}^{3} \times \mathbb{R}^{3}} d x d y \frac{\mathrm{M}(x) \mathrm{M}(y)}{|x-y|^{2}}<+\infty
$$

where $\mathrm{M}(x):=\sup _{t \in[0, T]}|V(x, t)|$.

$\left(\mathrm{R}^{\prime}\right)$ The function $t \mapsto V(x, t)$ is differentiable on $[0, T]$ for almost every $x$ and we have

$$
\int_{\mathbb{R}^{3} \times \mathbb{R}^{3}} d x d y \frac{\mathrm{N}(x) \mathrm{N}(y)}{|x-y|^{2}}<+\infty
$$

where $\mathbf{N}(x):=\sup _{t \in[0, T]}\left|\frac{\partial V(x, t)}{\partial t}\right|$.

Under these conditions we have indeed the following result.

Proposition 2. Assume that Hypotheses (R), ( $\left.R^{\prime}\right),(F)$ and (20) are valid. Then there exists a unique unitary evolution system $U_{H_{0}+V}(t, s)_{s, t \in[0, T]}$ on $L^{2}\left(\mathbb{R}^{3}\right)$ associated with the above realization of (76), for which all the conclusions of the corollary hold true.

Proof. Here we choose the Sobolev norm

$$
\|v\|_{+}=\left(\int_{\mathbb{R}^{3}} d x|\nabla v(x)|^{2}\right)^{\frac{1}{2}}
$$

for the fixed norm on $\mathcal{H}_{Q}=W^{1,2}\left(\mathbb{R}^{3}\right)$, while we have $\mathcal{H}_{Q}^{*}=W^{-1,2}\left(\mathbb{R}^{3}\right)$ for the corresponding adjoint space. Relation (57) is then valid for the Hermitian sesquilinear form $Q_{0}$ associated with $H_{0}=-\triangle_{x}+\kappa$, so that it is sufficient to prove that $(\mathrm{V})$ and $\left(\mathrm{V}^{\prime}\right)$ hold. From a simple adaptation of the proof of Theorem I.21 in [21] to the time-dependent case we can first infer that Hypothesis (R) implies $(\mathrm{V})$, where $Q_{V}(t)$ is the Hermitian sesquilinear form associated with the self-adjoint operator in $L^{2}\left(\mathbb{R}^{3}\right)$ corresponding to the multiplication by $V(x, t)$. The crucial point of this part of the argument is that the assumed uniformity in $t$ implies the time independence of the constants in $(\mathrm{V})$. 
In a similar way we claim that Hypothesis $\left(\mathrm{R}^{\prime}\right)$ implies $\left(\mathrm{V}^{\prime}\right)$. Indeed, since

$$
\begin{aligned}
& Q_{V}(t)[v, w] \\
& =\langle\mathrm{V}(t) v, w\rangle_{*}=\int_{\mathbb{R}^{3}} d x V(x, t) v(x) \bar{w}(x)
\end{aligned}
$$

for all $v, w \in W^{1,2}\left(\mathbb{R}^{3}\right)$ where $\langle., .\rangle_{*}$ denotes the duality bracket between $W^{1,2}\left(\mathbb{R}^{3}\right)$ and $W^{-1,2}\left(\mathbb{R}^{3}\right)$, we conclude from $\left(\mathrm{R}^{\prime}\right)$ and dominated convergence that the function $t \mapsto\langle\mathrm{V}(t) v, w\rangle_{*}$ is differentiable on $[0, T]$ with

$$
\begin{aligned}
& \frac{d}{d t}\langle\mathrm{~V}(t) v, w\rangle_{*} \\
& =\left\langle\mathrm{V}^{\prime}(t) v, w\right\rangle_{*}=\int_{\mathbb{R}^{3}} d x \frac{\partial V(x, t)}{\partial t} v(x) \bar{w}(x)
\end{aligned}
$$

since $x \rightarrow \mathrm{N}(x) v(x) \bar{w}(x) \in L^{1}\left(\mathbb{R}^{3}\right)$. Moreover, as is the case for $\mathrm{V}(t)$ the operator $\bigvee^{\prime}(t)$ is linear and bounded from $W^{1,2}\left(\mathbb{R}^{3}\right)$ into $W^{-1,2}\left(\mathbb{R}^{3}\right)$ and satisfies (29). Therefore, there does exist a unique unitary evolution system $U_{H_{0}+V}(t, s)_{s, t \in[0, T]}$ on $L^{2}\left(\mathbb{R}^{3}\right)$ such that all the stated conclusions hold true.

REMARKs. (1) It is plain that any kind of conditions other than (R) and $\left(\mathrm{R}^{\prime}\right)$ which imply the validity of $(\mathrm{V})$ and $\left(\mathrm{V}^{\prime}\right)$ will lead to the same statement as that of the proposition.

(2) Since the operators $U_{H_{0}+V}(t, s)$ are related to the operators $U_{-\triangle+V}(t, s)$ associated with the solution to the initial-value problem

$$
\begin{aligned}
i \frac{\partial u(x, t)}{\partial t} & =\left(-\triangle_{x}+V(x, t)\right) u(x, t), \quad(x, t) \in \mathbb{R}^{3} \times(s, T], \\
u(x, s) & =v(x), \quad x \in \mathbb{R}^{3},
\end{aligned}
$$

by

$$
U_{H_{0}+V}(t, s)=e^{-i \kappa(t-s)} U_{-\triangle+V}(t, s),
$$

it is immediate that a result similar to that of Proposition 2 holds for $U_{-\triangle+V}(t, s)$. The corresponding approximating functions simply differ by at most a trivial factor of modulus one.

Acknowledgments. The first author would like to take this opportunity to thank the Forschungsinstitut für Mathematik of the ETH in Zurich and the Complexo Interdisciplinar da Universidade de Lisboa in Lisbon for their very kind hospitality and their generous financial support during the period leading up to the completion of this work. He is particularly indebted to Professors T. Rivière and J.-C. Zambrini for having made his visits possible.

\section{References}

[1] Adams, R. A., Fournier, J. J. F., Sobolev Spaces, Academic Press (2003), New York. 
[2] Albeverio, S., Gesztesy, F., Hoegh-Krohn, R., Holden, H., Solvable Models in Quantum Mechanics, Springer Verlag (1988), New York.

[3] Chernoff, P. R., Note on Product Formulas for Operator Semigroups, Journal of Functional Analysis 2 (1968) 238-242.

[4] Chernoff, P. R., Semigroup Product Formulas and Addition of Unbounded Operators, Bulletin of the American Mathematical Society 76 (1970) 395-398.

[5] Chernoff, P. R., Product Formulas, Nonlinear Semigroups, and Addition of Unbounded Operators, Memoirs of the American Mathematical Society 140 (1974), Providence.

[6] Chorin, A. J., Hughes, T. J. R., McCracken, M. F., Marsden, J. E., Product Formulas and Numerical Algorithms, Communications on Pure and Applied Mathematics 31 (1978) 205-256.

[7] Davies, E. B., One-Parameter Semigroups, Academic Press (1980), London.

[8] Dell' Antonio, G. F., Figari, R., Teta, A., The Schrödinger Equation with Moving Point Interactions in Three Dimensions, Canadian Mathematical Society Conference Proceedings 28 (2000) 99-113.

[9] J. Dimock, $P(\varphi)_{2}$-Models with Variable Coefficients, Annals of Physics 154 (1984) 283-307.

[10] Faris, W. G., The Product Formula for Semigroups defined by Friedrichs Extensions, Pacific Journal of Mathematics 22 (1967) 47-70.

[11] Faris, W. G., Product Formulas for Perturbations of Linear Propagators, Journal of Functional Analysis 1 (1967) 93-108.

[12] Ichinose, T., Tamura, H., Error Estimate in Operator Norm of Exponential Product Formulas for Propagators of Parabolic Evolution Equations, Osaka Journal of Mathematics 35 (1998) 751-770.

[13] Kato, T., Trotter's Product Formula for an Arbitrary Pair of Self-Adjoint Contraction Semigroups, in: Topics in Functional Analysis (I. Gohberg and M. Kac, editors) (1978) 185-195, Academic Press, New York.

[14] Kato, T., Perturbation Theory for Linear Operators, Die Grundlehren der mathematischen Wissenschaften 132 (1984) Springer Verlag, New York.

[15] Kisyński, J., Sur les Opérateurs de Green des Problèmes de Cauchy Abstraits, Studia Mathematica 23 (1964) 285-328.

[16] Lions, J. L., Équations Différentielles Opérationnelles et Problèmes aux Limites, Die Grundlehren der mathematischen Wissenschaften in Einzeldarstellungen 111 (1961) Springer Verlag, Berlin. 
[17] Neidhardt, H., Zagrebnov, V. A., Trotter-Kato Product Formula and Operator-Norm Convergence, Communications in Mathematical Physics 205 (1999) 129-159.

[18] Neidhardt, H., Zagrebnov, V. A., Linear Non-Autonomous Cauchy Problems and Evolution Semigroups, Advances in Differential Equations 14 (2009) 289-340.

[19] Posilicano, A., The Schrödinger Equation with a Moving Point Interaction in Three Dimensions, Proceedings of the American Mathematical Society 135 (2007) 1785-1793.

[20] Reed, M., Simon, B., Methods of Modern Mathematical Physics, II (1975) Academic Press, London.

[21] Simon, B., Quantum Mechanics for Hamiltonians Defined as Quadratic Forms, Princeton Series in Physics (1971) Princeton University Press.

[22] Tanabe, H., Equations of Evolution, Monographs and Studies in Mathematics 6 (1979) Pitman, London.

[23] Trotter, H., On the Product of Semigroups of Operators, Proceedings of the American Mathematical Society 10 (1959) 545-551.

[24] Vuillermot, P.-A., Wreszinski, W. F., Zagrebnov, V. A., A General Trotter-Kato Formula for a Class of Evolution Operators, Journal of Functional Analysis 257 (2009) 2246-2290.

[25] Vulllermot, P.-A., A Generalization of Chernoff's Product Formula for Time-Dependent Operators, Journal of Functional Analysis 259 (2010) 2923-2938.

[26] Yosida, K., Functional Analysis, Classics in Mathematics Series (1995) Springer Verlag, New York. 